\title{
Riesenwuchs auf verrotteten Halmen von Scirpus lacustris $\mathrm{L}$.
}

\author{
von K. SEIDEL
}

(Hydrobiologische Anstalt der Max-Planck-Gesellschaft in Plön)

Während die abgestorbenen Halme von Phragmites communis über Winter, oft sogar bis in den Frühsommer, an der Pflanze verbleiben und nach der Loslösung vom Rhizom sehr schwer verrotten, verhält sich Scirpus lacustris L. darin gänzlich anders: Die abgestorbenen Halme oder auch nur die abgestorbenen Halmteile werden über Winter von der Pflanze durch Sturm oder Eisbildung getrennt. Da sie ein sehr geringes spezifisches Gewicht haben, setzt sie der Wellenschlag als ,Treibsel" am Ufer ab und dort zerfallen sie in wenigen Monaten. Sie werden dann wie kleine Papierfetzen vom Wind über die Weidenkoppeln getrieben. Setzt man jedoch die abgestorbenen Halme zu Komposthaufen auf, so verrotten sie sehr schnell und bilden ein lockere, humose, sehr wohlriechende Erde. Wir haben diese Kompostbereitung seit 1947 systematisch durchgeführt und sie seit 1949 für Saat- und Pflanzversuche verwandt.

Es wurden in dieser Erde Aussaaten von Stauden, Sommerblumen und von anspruchsvollen Gartengemüsen gemacht und die Sämlinge darin weiter kultiviert. Das vegetative und generative Wachstum war auffallend üppig; die Blütenfarben z.B. bei Aster alpinus Primula veris und Trollius europaeus waren tiefer und leuchtender als die der Vergleichspflanzen. Während aber das Blattwachstum bei Tropaeolum maius in dieser Erde besonders üppig war, zeigten die Blüten keinerlei Veränderungen. Hingegen waren bei Tagetes erecta Blätter und Blüten auffällend groß.

Die beigefügten Fotos verdeutlichen die auffallenden Größenunterschiede im Blattwachstum bei Tropaeolum maius (Abb. 1) und im Blatt- und Blütenwachstum bei Tagetes erecta (Abb. 2).

$$
\text { Er } 1 \text { äuterung } z \text { u A b b. 1: }
$$

Bezeichnung: links und rechts Riesenformen der Blätter von Tropaeolum maius (a-Formen).

In der Mitte: Normale Form (n-Formen). Standort: Klostergarten Preetz/Holstein. 
Erde: n-Formen: Gartenerde (humoser Lehmboden) a-Formen: Kompost aus Halmen von Scirpus lacustris.

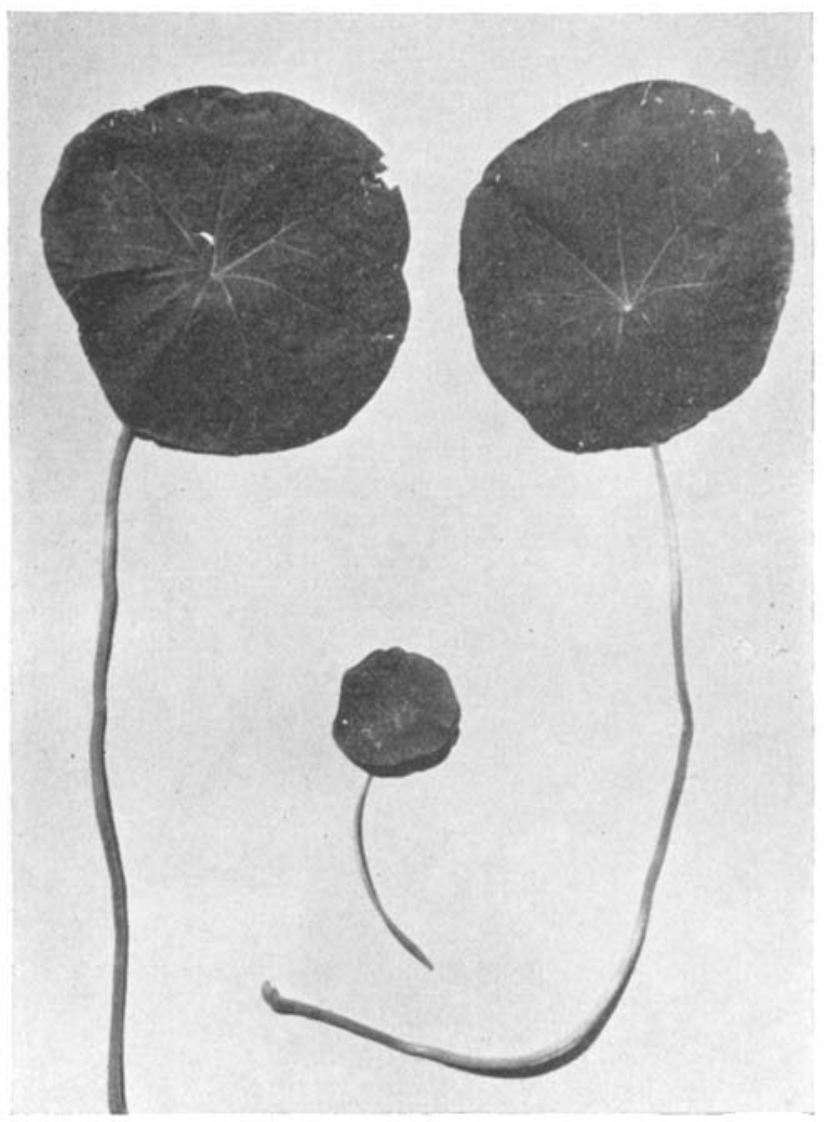

Abb. I

Entfernung der beiden Standorte voneinander: $5 \mathrm{~m}$. Aussaat am: 1. Mai 1951.

Gemessen am: 4. Oktober 1951.

\begin{tabular}{lccc} 
& \multicolumn{3}{c}{ Blatt } \\
\hline & links & Mitte & rechts \\
\hline Stiellänge in cm & 51,4 & 19,2 & $\left.64,2^{1}\right)$ \\
Stieldicke in mm, Basis & 9,0 & 2,9 & 11,7 \\
Stieldicke in mm, Spitze & 3,8 & 1,9 & 3,9 \\
Blattbreite in cm & 18,2 & 6,6 & 19,4 \\
Blattfläche in qcm & 260,023 & 34,197 & 295,442
\end{tabular}

1) Im I2, II-I952 würden Stiellängen bis $70,2 \mathrm{~cm}$ gemessen. 
Erläuterung zu A b b. 2:

Bezeichnung: links Riesenform von Tagetes erecta rechts normale Form von Tagetes erecta.

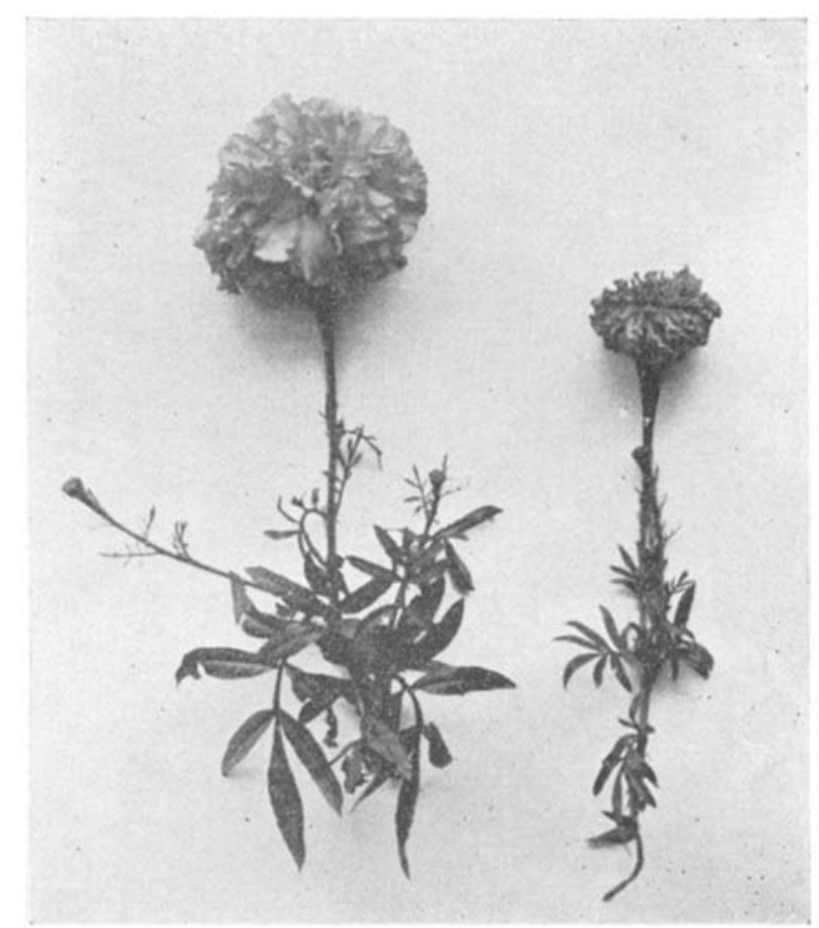

Abb. 2

Standort: Klostergarten Preetz/Holstein.

Erde: n-Formen: humoser Lehmboden; a-Formen: Kompost von Sc. lac.

Entfernung der beiden Standorte voneinander: $2 \mathrm{~m}$.

Ausgepflanzt am: 2. Juni 1951.

Gemessen am: 4. Oktober 1951.

In den folgenden Jahren wiederholten wir die Versuche und es seien hier auffallende Merkmale von Tropaeolum maius wiedergegeben:

Der B 1 a t t s t i e 1 ist unifacial mit Dorsal- und Ventralmedianus (Troll 1948, S. 316). Er weist aber bei den a-Formen eine kolbenförmige Verdickung an der Stielbasis und eine Zuspitzung am Übergang zur Blattspreite auf. Diese Veränderungen konnten bei den n-Formen nirgends beobachtet werden (Abb. 3). 
Die Epidermis des Blattstieles ist bei den a-Formen stark verändert. In vielen untersuchten Exemplaren sind die Zellen bedeutend kleiner, sind aber in viel größerer Anzahl vorhanden. In

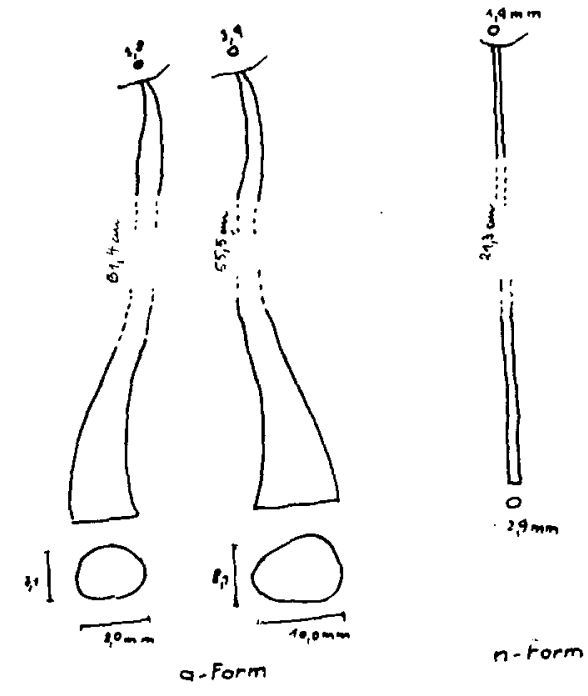

Abb. 3

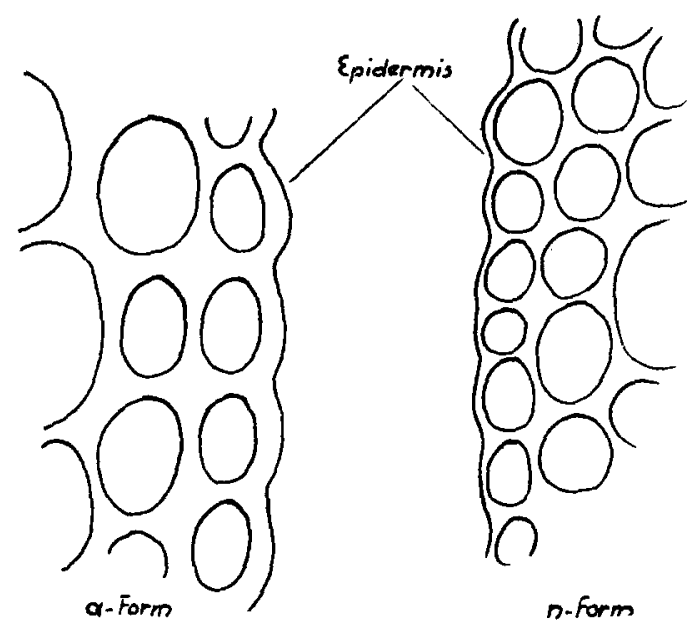

Abb. 4

allen Fällen waren bei den a-Formen die Zellwände der Epidermis sehr stark verdickt. Das sich anschließende Parenchym zeigte bei den a-Formen eine größere Regelmäßigkeit als bei den $\mathrm{n}$-Formen (Abb. 4). 
Die $\mathrm{L}$ a $\mathrm{m}$ in a hat in beiden Fällen die Schildform mit nahezu zentralen Stielenansatz. Abnorme Blattbildungen, wie sie bei Tropaeolum maius oft beobachtet werden (siehe auch HEGI IV/3 S. 1729) entwickelten sie in keinem Fall. Die Anzahl der fast radialen Hauptnerven ist trotz des erheblichen Größenunterschiedes der a- und nFormen nicht unterschiedlich. Es muß sich also das intercostale Spreitenbereich stark auswachsen. In der Dicke der Lamina konnte bei beiden Formen kein Unterschied festgestellt werden. Die in Größe und Gestalt sehr unterschiedlichen Blatthaare wurden in beiden Fällen gefunden (Abb. 5a und $5 \mathrm{~b}$ ). Während bei einigen

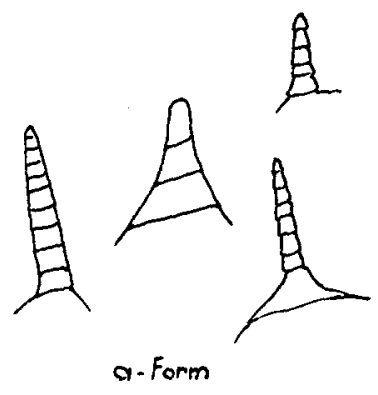

Abb. $5 a$

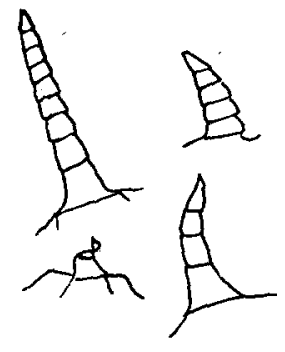

n-Form

Abb. $5 b$

Messungen die Anzahl der Blatthaare bei den a-Formen stark überwogen, waren es bei anderen Messungen gerade die geringe Anzahl der Haare, die bei diesen Formen auffielen.

Das üppige vegetative Wachstum aber auch die auffallende generative Entwicklung bei Pflanzen vom Scirpus-lacustris-Humus, veranlassten uns, die Erde zu untersuchen ${ }^{1}$ ).

Es liegen bisher folgende Analysen vor: (Unters. 17.6.52)

Phosphorsäure $\left(\mathrm{P}_{2} \mathrm{O}_{5}\right.$ wurzellöslich nach Neubauer

$\mathrm{mg}$ in $100 \mathrm{~g}$ Boden $\ldots \ldots \ldots \ldots \ldots \ldots \ldots \ldots$ 20,4

Kali $\left(\mathrm{K}_{2} \mathrm{O}\right)$ wurzellöslich nach Neubeuer

$\mathrm{mg}$ in $100 \mathrm{~g}$ Boden ................ 57,0

Kalk (Anfangs-pH-Zahl (Säurezahl)

in $\mathrm{n}-\mathrm{KCl}$ Aufschlämmung $\ldots \ldots \ldots \ldots \ldots \ldots \ldots, 7,0$

Stickstoff nach Hasenbäumer $\ldots \ldots \ldots \ldots \ldots \ldots \ldots$ 12,3 mg

Gesamt-Kupfer $(\mathrm{Cu}) \ldots \ldots \ldots \ldots \ldots \ldots \ldots \ldots .90 \quad 90$ ppm

Austauschbares Mangan (sofort verfügbares Mangan) $47,4 \mathrm{ppm}$

Leicht reduzierbares Mangan (in Kürze unter Mit

wirkung biochemischer Faktoren verfügb. Res. .. 119,6 ppm

1) Ich verdanke diese Untersuchungen der Zusammenarbeit mit Frl. Dr. BoNIG, Landw. Unters.- u. Forschungsanstalt Kiel. 
Zum Vergleich seien Neubauer-Zahlen für Phosphorsäure und Kali, Landw. Versuchs- und Forschungsanstalt Augustenberg, angegeben:

$$
\begin{array}{cc}
\begin{array}{c}
\mathrm{mg} \mathrm{P}_{2} \mathrm{O}_{5} / 100 \mathrm{~g} \\
\text { Boden }
\end{array} & \begin{array}{c}
\mathrm{mg} \mathrm{K}_{2} \mathrm{O} / 100 \mathrm{~g} \\
\text { Boden }
\end{array} \\
\hline 5,0-69 & 15,0-19,9 \\
6,0-79 & 22,0-29,9 \\
7,0-8,9 & 28,0-39,9
\end{array}
$$

guter Sandboden

guter sandiger Lehmboden

guter toniger Lehmboden

Auffallend hoch ist der Anteil der Spurenelemente Kupfer und Mangan in dieser Erde aus verrotteten Scirpus-lacustris-Halmen. Die Untersuchungen werden fortgesetzt.

\section{Literatur.}

Hegi „Illustrierte Flora von Mitteleuropa" München 1906-1931

Troll „Allgemeine Botanik” Stuttgart 1948. 